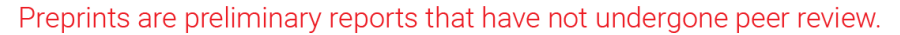 They should not be considered conclusive, used to inform clinical practice, or referenced by the media as validated information. \\ Innovation and inheritance: A novel strategy for the development of anti-yellowing cosmetic material from TCM formula
}

\section{Shiyi WANG}

Beijing Technology and Business University

\section{Hong MENG}

Beijing Technology and Business University

\section{Yinmao DONG}

Beijing Technology and Business University

Li LI

Beijing Technology and Business University

\section{Tuya BAO}

Beijing University of Chinese Medicine

\section{Fan YI ( $\square$ fantasyee@btbu.edu.cn )}

Beijing Technology and Business University https://orcid.org/0000-0002-7476-616X

\section{Research}

Keywords: TCM formula, anti-yellowing combination; hierarchical clustering analysis, skincare

Posted Date: May 15th, 2020

DOI: https://doi.org/10.21203/rs.3.rs-27852/v1

License: (9) (1) This work is licensed under a Creative Commons Attribution 4.0 International License. Read Full License 


\section{Abstract}

Background Yellow complexion is a major skin issue that bothers Chinese women. The major factor leading to sallow complexion is the accumulation of advanced glycosylation end products (AGEs). Traditional Chinese medicine (TCM) treats dull, yellow complexion as being associated with liver-, spleen- or stomach-related functional disorders. Through analysis of prescription data, we explored combinations of new prescriptions with yellow-dispelling effects.

Methods We extracted the most commonly used drug pairs and core medicine in anti-yellowing formulas, then carry out hierarchical clustering analysis and extract new formula combinations. The combination effect was determined by falvnoe, DPPH radical scavenging activity and Non-enzymatic glycosylation inhibiting activity. Toxicological analysis included HET-CAM, ЗT3 phototoxicity, multiple irritation and patch test.

Result Three empirical formulas were identified according to drug frequency analysis, and a new formula was identified according to the results of hierarchical clustering analysis. A series of biochemical experiments proved that the best anti-yellow-complexion formula combination was the new one. The results of the safety investigation showed that the new formula at a concentration of $5 \%$ had no eye irritation effects, no irritant or corrosive effects on mammalian skin, and no adverse reactions with human skin.

Conclusion This study explored and analysed the internal laws of TCM for treatment of yellow complexion and, on this basis, developed a plant combination extract material with an anti-yellowing effect on skin.

\section{Background}

Since 2015, China has vigorously supported the development of traditional Chinese medicine (TCM) and has issued nearly 20 national policies to promote national-level strategies for this purpose. According to statistics from Euromonitor, China's domestic cosmetics market reached 221.072 billion yuan in 2017, with a year-overyear growth of $11.9 \%$ (YoY + 5.5 PCT), and has become the second-largest cosmetics market in the world. Compared with the mature Japanese market, it is still too early for China's cosmetics market to hit the ceiling, and the industry certainly has prospects for long-term development. In the past decade, TCM, with its core ingredients, has emerged as a domestic brand in China, and TCM products have received a good market response, gradually becoming the pillar products of pharmaceutical companies. Thus, TCM products are not only suitable for drug development but also exhibit excellent performance in beauty and personal care products.

The application of TCM in the cosmetics industry has the following characteristics: (1) Medicinal plant resources are abundant, and there are more than 300 kinds of plant TCM products included in the 2015 edition of the "list of used cosmetics raw materials" and "Chinese pharmacopeia", which are receiving increasing attention. Many famous local brands use TCM products as the core active ingredients. (2) Several ancient Chinese philosophies, such as yin-yang and the five elements, or specific types of individual plants are used as marketing points, and few formulations are constructed under the guidance of theoretical thoughts such as prescription medication in TCM. There is market potential for the development of such products. (3) Drug research and development primarily focuses on efficacy, while cosmetic development should focus first on safety and then consider efficacy. Both products are heterogenous, and the list of used raw materials should be 
included with the cosmetics developed. (4) As raw materials for cosmetics, TCMs has broad prospects and considerable market value.

Dullness, lack of transparency, and yellowish complexion are the top keywords associated with Chinese women's awareness of skin problems. Dark yellow complexion is caused by three main factors: (1) Sebum ageing: When the cuticle is dry, the sebum level is low, and the scales produce an irregular surface, reducing the specular reflection of light, so the skin takes on a dark matte appearance. Sebum is removed by cleaning and other means before significant amounts of squalene degrade to form a yellow polar compound residue, causing a dark yellow appearance ${ }^{[1]}$. (2) Food and medicines containing yellow dye and pathological jaundice ${ }^{[2]}$. Cholecystitis causes yellowing ${ }^{[3]}$, carotenoids are orange, and melanin is brown. Excessive intake of foods such as oranges and sea buckthorn can lead to temporary yellowing of the skin ${ }^{[4]}$. This can also be caused by a heterogeneous population of keratin, elastin and connective tissue; lipid metabolism; and abnormal metabolic, inflammatory, or organ dysfunction states ${ }^{[5]}$. (3) Accumulation of advanced glycosylation end products (AGEs): AGEs in the skin emit characteristic fluorescence with a wavelength of $330-500 \mathrm{~nm}$, which is commonly observed in diabetes research ${ }^{[6]}$. Dyer ${ }^{[7]}$ found that non-enzymatic glycation reactions of collagen and elastin molecules occurred, and the number of AGEs increased with age, leading to yellowing, darkening and decreased visual clarity of the skin. The darkening of the skin has been quantified and graded $^{[8,9]}$. Ageing sebum can be removed by cleaning, and dietary and pathological factors can be avoided, so AGEs are the leading cause of dark yellow facial skin.

Additionally, AGEs can accelerate skin ageing ${ }^{[10-14]}$, affect skin barrier function ${ }^{[15-19]}$, block skin microcirculation $^{[20]}$, and aggravate pigment deposition ${ }^{[7,21-23]}$, which can affect the overall health and beauty of the skin.

TCM has a long history of hair care; it originated in the Qin and Han dynasties, formed in the Sui and Tang dynasties, developed in the Song and Jin dynasties, and was enriched in the Ming and Qing dynasties. Several medicines were developed during these past regimes, and nearly 2,000 TCM hair care formulas were recorded. However, the medicines prescribed by different doctors are affected by the time, climate, geographical location, and specific conditions of patients, and the dosage and compatibility of prescriptions vary. Therefore, the use of new combinations to analyse the data for historical prescriptions and discover the underlying fundamental rules for combination using the methods and technologies of TCM is a scientific process. In this study, a new plant composition suitable for use as an effective raw material in cosmetics was proposed based on a classic Chinese medicinal composition, and the methodology for the development of this form was preliminarily explored.

Based on TCM principles, dull yellow complexion is associated with liver-, spleen- or stomach-related functional disorders. Through data-based analysis of formulas, we explored the a new formula combination with a yellowdispelling effect.

\section{Method And Material}

\subsection{Formula retrieval, screening and proofreading}




\subsubsection{Formula retrieval}

The database used was a Chinese traditional medicine database (Chinese Academy of Chinese Medical Sciences). Two kinds of search words were used according to the description of yellow complexion in TCM classics. The first word described the appearance directly, and the indirect description was based on textual meaning and pathogenesis. There were 7 search terms used for the first round of the search.

\subsubsection{Formula screening}

We found that some formulas unsuitable as cosmetics or for the treatment of yellow complexion needed to be removed. These were summarized into the following five categories:

(1) Associated with severe skin diseases;

(2) Associated with short-term body function disorder caused by alcohol abuse or parasites;

(3) People in special physiological stages, such as childhood and pregnancy;

(4) People who were sallow and emaciated because of major diseases;

(5) Retrieved formula was not associated with the yellowing of facial skin.

We also made restrictions on the medicinal materials that constituted the formulas. The rules were as follows:

(1) Removal of formulas that contained materials that are not included in the Chinese Pharmacopoeia and had no better alternatives.

(2) Removal of formulas that contained fossils and rare animals. If widely recognized substitutes were available, the formula could be retained.

\subsubsection{Formula proofreading}

After retrieval and screening,the data were audited, integrated and classified. The names of the formulas were checked to ensure that there are no different formulas with the same name and that the same formula was not designate different names. With changes in time, place of origin and accent, the names of medicinal materials may have changed. The standard for Chinese medicinal synonymy in this study was first compiled according to the 2015 edition of the Pharmacopoeia of the People's Republic of China and then according to the customary names of modern Chinese medicines. A comparison of the ancient and modern names of medicinal materials is shown in Table 1. 
Table 1

Comparison of the ancient and modern names of herbal medicines

\begin{tabular}{|c|c|c|c|c|}
\hline NO. & Scientific Name & TCM Name & $\begin{array}{l}\text { Chinese } \\
\text { Pharmacopoeia }\end{array}$ & $\begin{array}{l}\text { Classic } \\
\text { Name }\end{array}$ \\
\hline 1 & Rehmannia glutinosa Libosch. & $\begin{array}{l}\text { Rehmanniae Radix } \\
\text { Praeparata }\end{array}$ & प्राप्त & 吅 \\
\hline 2 & Rehmannia glutinosa Libosch. & Rehmanniae Radix & प्राप्त & प्स \\
\hline 3 & $\begin{array}{l}\text { Ophiopogon japonicus (L. f) } \\
\text { Ker-Gawl. }\end{array}$ & Ophiopogonis Radix & 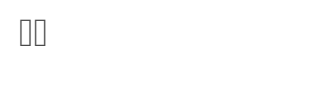 & प्स \\
\hline 4 & Areca catechu L. & Arecae Semen & 0 & वस्त \\
\hline 5 & Curcuma phaeocaulis Val. & Curcumae Rhizoma & 吅 & प्राप्या \\
\hline 6 & Glycyrrhiza uralensis Fisch. & $\begin{array}{l}\text { Glycyrrhizae Radix Et } \\
\text { Rhizoma }\end{array}$ & 吅 & 吅 \\
\hline 7 & Dioscorea opposita Thunb. & Dioscoreae Rhizoma & 吅 & 吅 \\
\hline 8 & Cinnamomum cassia Presl & Cinnamomi Cortex & 吅 & 怄, 讴 \\
\hline 9 & Angelica sinensis (Oliv.) Diels & Angelicae Sinensis Radix & 吅 & 吅 \\
\hline 10 & $\begin{array}{l}\text { Atractylodes macrocephala } \\
\text { Koidz. }\end{array}$ & $\begin{array}{l}\text { Atractylodis Macrocephalae } \\
\text { Rhizoma }\end{array}$ & $\mathrm{at}$ & 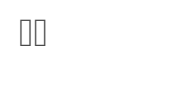 \\
\hline 11 & Citrus reticulata Blanco & Citri Reticulatae Pericarpium & 吅 & 吅 \\
\hline 12 & Psoralea corylifolia L. & Psoraleae Fructus & वस्प & प्राप्त \\
\hline 13 & $\begin{array}{l}\text { Polygonatum odoratum } \\
\text { (Mill.)Druce }\end{array}$ & Polygonati Odorati Rhizoma & 吅 & 吅 \\
\hline 14 & $\begin{array}{l}\text { Sparganium stoloniferum Buch. } \\
\text {-Ham. }\end{array}$ & Sparganii Rhizoma & 吅 & 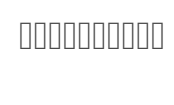 \\
\hline 15 & $\begin{array}{l}\text { Astragalus membranaceus } \\
\text { (Fisch. ) Bge }\end{array}$ & Astragali Radix & 吅 & 吅 \\
\hline 16 & Ligusticum chuanxiong Hort. & Chuanxiong Rhizoma & 吅 & प \\
\hline 17 & Poria $\operatorname{cocos}$ (Schw.)Wolf & Poria & 吅 & प्स \\
\hline 18 & Cinnamomum cassia Presl & Cinnamomi Ramulus & 吅 & प \\
\hline 19 & Schizonepeta tenusfolia Briq. & Schizonepetae Spica & प्राप्त & प्राप्त \\
\hline 20 & Codonopsis tangshen Oliv. & Codonopsis Radix & 吅 & 吅 \\
\hline 21 & $\begin{array}{l}\text { Magnolia officinalis Rehd.et } \\
\text { Wils. }\end{array}$ & Magnoliae Officinalis Cortex & 吅 & 吅 \\
\hline 22 & Cyperus rotundus $\mathrm{L}$. & Cyperi Rhizoma & 吅 & प्राप्त \\
\hline 23 & Nelumbo nucifera Gaertn. & Nelumbinis Semen & 0 & 0 \\
\hline 24 & $\begin{array}{l}\text { Zanthoxylum bungeanum } \\
\text { Maxim. }\end{array}$ & Zanthoxyli Pericarpium & 吅 & 吅 \\
\hline
\end{tabular}




\begin{tabular}{|lllll|}
\hline NO. & Scientific Name & TCM Name & $\begin{array}{l}\text { Chinese } \\
\text { Pharmacopoeia }\end{array}$ & $\begin{array}{l}\text { Classic } \\
\text { Name }\end{array}$ \\
\hline 25 & Cistanche deserticola Y.C.Ma & Cistanches Herba & 맘 & \\
\hline
\end{tabular}

Table 1. Comparison of the ancient and modern names of herbal medicines(additional file)

The name, alias, presence, source, preparation, usage and dosage of the formulas were recorded.

\subsection{Analysis of formulas}

We used the TCM Inheritance Auxiliary System ( $v$ 2.5) for formula analysis, which was developed by the Institute of Chinese Materia Medica, China Academy of Chinese Medical Sciences. The 84 formulas were entered into the system and reviewed by 3 people. The high-frequency drugs and drug combinations in the formulas could be determined by using the software. Then, the compatibility rules of the formulas were summarized, and datamining algorithms were used to obtain new formulas based on the formula data set.

\subsection{Biochemical efficacy test}

\subsubsection{Chemicals and reagents}

Sodium nitrite, aluminium nitrate, sodium hydroxide, rutin, absolute ethyl alcohol, 2,2-diphenyl-1-picrylhydrazyl (DPPH), ascorbic acid, bovine serum albumin (BSA), glucose, phosphate-buffered saline (PBS), aminoguanidine, sodium dodecyl sulphate (SDS), and chlorpromazine hydrochloride (CPZ) were used.

\subsubsection{Extract Preparation}

All the herbs were crushed sequentially and passed through a 10-mesh sieve. Empirical formulas were mixed according to the ratios recorded in the classics, and the new formula was mixed with equal proportions. The material-liquid ratio was 1:15; the mixture was soaked for 1 hour and then placed at $80^{\circ} \mathrm{C}$ for 2 hours. The extracts were filtered through $0.45-\mu \mathrm{m}$ pore size filter paper and freeze-dried for 24 hours. Finally, we obtained the formula as a lyophilized powder.

\subsubsection{Test for flavone}

Sodium nitrite-aluminium nitrate-sodium hydroxide colorimetry was used to precisely weigh $0.1 \mathrm{~g}$ of the rutin reference substance and dissolve it in $75 \%$ ethanol in a $100-\mathrm{ml}$ volumetric bottle with a constant volume and a solution mass concentration of $1.0 \mathrm{~g} / \mathrm{L}$. Gradient dilution of the rutin reference substance solution was performed serially with $4 \mathrm{~mL}$ of deionized water. Then, $0.3 \mathrm{~mL}$ of a $5 \%$ (mass fraction) aqueous solution of sodium nitrite was added. After $5 \mathrm{~min}, 0.3 \mathrm{~mL}$ of a $10 \%$ (mass fraction) aluminium nitrate aqueous solution was added. Then, $2 \mathrm{~mL}$ was taken for a quality score, and after $6 \mathrm{~min}, 1 \mathrm{~mol} / \mathrm{L}$ sodium hydroxide aqueous solution and $2.4 \mathrm{~mL}$ of deionized water was added, and after $10 \mathrm{~min}$, the absorbance at $510 \mathrm{~nm}$ was determined, with $75 \%$ ethanol as the control group. The ordinate was used as the absorbance value, and the concentration of the rutin reference substance was used as the abscissa to prepare a standard curve. The solution was prepared by using the freeze-dried powder and deionized water. The absorbance was measured according to the above steps, and the total flavonoid concentration of the redissolved liquid was calculated according to the standard curve equation. 
Standard curve: absorbance $=0.7099 x-0.0135 R^{2}=0.9996$ ( $y$ value is absorbance; $x$ value is additive mass concentration).

\subsubsection{DPPH radical scavenging activity}

The antioxidant activity of DPPH determined based on the scavenging ability of DPPH free radicals. DPPH ethanol solution $(2 \times 10-4 \mathrm{~mol} / \mathrm{L}), 0.01 \%$ vitamin $\mathrm{C}$ aqueous solution as the positive control, distilled water as the negative control, and anhydrous ethanol as the control group were used. In tube A, the sample solution was mixed with an equal volume of DPPH solution; in tube $B$, equal volumes of anhydrous ethanol and DPPH solution were mixed; in tube $\mathrm{C}$, the sample solution was mixed with an equal volume of anhydrous ethanol. After the 30-min light-protected reaction, the absorbance of each tube was measured at $517 \mathrm{~nm}$.

$\%_{\text {inhibition }}=[(B+C)-A] / B * 100 \%$

\subsubsection{Non-enzymatic glycosylation inhibiting activity}

High-pressure steam sterilization experiment supplies and solvents were prepared before the experiment. The reaction solution contained $50 \mathrm{~mL}$ of PBS, $0.5 \mathrm{~g}$ of bovine serum protein, and $2.7 \mathrm{~g}$ of glucose; the sample solution was prepared in sterilized water with gradient dilutions to $2 \%, 1 \%, 0.5 \%$; the positive control group was made of aminoguanidine hydrochloride in sterilized water, diluted to $2 \%, 1 \%$ and $0.5 \%$. All the solutions were filtered and sterilized with a $0.2 \mu \mathrm{m}$ filter membrane. The incubation was performed at $37^{\circ} \mathrm{C}$ for 7 days without light, and then, the fluorescence intensity was detected at an excitation wavelength of $370 \mathrm{~nm}$ and an emission wavelength of $440 \mathrm{~nm}$.

$\%_{\text {inhibition }}=[1-(\mathrm{A} 1 \mathrm{~A} 2) / \mathrm{A} 0] \times 100 \%$

\subsection{Process optimization 2.4.1 Single factor experiment}

The three factors ethanol concentration, extraction time and solid-liquid ratio were optimized with the total flavonoid content as the reference index. For optimization of ethanol concentration, we used a uniform solidliquid ratio of $1: 30$, ultrasonic extraction time of $30 \mathrm{~min}$, and ethanol volume fraction of $0 \%$ (pure water), $60 \%$, or $90 \%$. For optimization of extraction time, the extraction solvent was $60 \%$ ethanol solution, the solid-liquid ratio was 1:30, and the ultrasonication time was $20 \mathrm{~min}, 30 \mathrm{~min}$, or $40 \mathrm{~min}$. For optimization of the solid-liquid ratio, the uniform solvent was $60 \%$ ethanol, the ultrasonic extraction time was $30 \mathrm{~min}$, and the solid-liquid ratios were 1:10, 1:20, 1:30, 1:40, and 1:50.

\subsubsection{Orthogonal test}

The total flavonoid content and the rate of inhibition of non-enzymatic glycosylation were used as reference indexes in the orthogonal experiment. The optimization conditions were as follows: the material-liquid ratios were 1:20, 1:30, and 1:40; the ultrasonic extraction times were $20 \mathrm{~min}, 30 \mathrm{~min}$, and $40 \mathrm{~min}$, and the ethanol volume fractions were $70 \%, 80 \%$, and $90 \%$. A orthogonal experiment with three factors and three levels was carried out.

\subsection{Safety tests}




\subsubsection{HET-CAM test}

The method reference was EURL-ECVAM DB-ALM Method Summary n96: Hen's Egg Test on the Chorioallantoic Membrane (HET-CAM). The positive control was $1 \%$ SDS, and the negative control was saline.

\subsubsection{In vitro 3T3 NRU phototoxicity test}

Section 18 of chapter VI of the Cosmetics Safety Technical Specification (2015 Edition) was referred to for the method for the in vitro 3T3 neutral red uptake phototoxicity test of chemical raw materials for cosmetics with Balb/c 3T3 fibroblasts. Chlorpromazine hydrochloride (CPZ) was used as a positive control.

\subsubsection{Multiple skin irritation test}

Section 4 of Chap. 7 of the Cosmetic Safety Technical Specification (2015 edition) was referred to for the skin irritation test method.

\subsubsection{4-hour human body closed patch test}

Section 2 of Chap. 7 of the Cosmetic Safety Technical Specification (2015 edition) was referred to for the human skin closed patch test method.

\section{Result}

3.1Formula retrieval and analysis

\subsubsection{Formula retrieal resultes}

After retrieval and screening, a total of 84 formulas based on maintenance supplements were obtained. Three were from the Tang period and earlier; 40 were from the Song, Jin and Yuan periods; 28 were from the Ming and Qing periods; and 12 were from the Ming and Qing periods.

\subsubsection{Medication rules of anti-yellow formulas}

The anti-yellowing formulas involved 173 TCMs. The top 20 medicines with the highest frequency and their frequencies are shown in Figure 1.

Figure 1. High-frequency TCMs in anti-yellowing formulas. Approximately half of the formulas contained four herbs: Indian bread (Poria), Ginseng, Atractylodes and Chinese angelica.

Slightly cold and very cold were included in the cold category, and slightly hot and very hot were included in the hot category. The samples were weighted by frequency, and then, the regularity of nature and flavour were analysed (Table 2-3)

Table2. Nature and flavor of medicines regularity of anti-yellow formulas 


\begin{tabular}{|c|c|c|c|}
\hline Nature and flavour & Four nature of drugs & Frequency $₫$ Times $₫$ & Rate $\nabla \% \bigotimes$ \\
\hline \multirow[t]{6}{*}{ Four nature } & Warm & 468 & 57.71 \\
\hline & Plain & 158 & 19.48 \\
\hline & Cold & 116 & 14.30 \\
\hline & Hot & 61 & 7.52 \\
\hline & Cool & 8 & 0.99 \\
\hline & Total & 811 & 100 \\
\hline \multirow[t]{7}{*}{ Five flavours } & Sweet & 469 & 35.61 \\
\hline & Pungent & 400 & 30.37 \\
\hline & Bitter & 338 & 25.66 \\
\hline & Sour & 70 & 5.32 \\
\hline & Astringent & 22 & 1.67 \\
\hline & Salty & 18 & 1.37 \\
\hline & Total & 1317 & 100 \\
\hline
\end{tabular}

Table 3. Channel tropism regularity of anti-yellow formula

\begin{tabular}{lll} 
Channel tropism & Frequency & Rate $\% \bigotimes$ \\
\hline Spleen & 543 & 24.23 \\
\hline Lung & 302 & 13.48 \\
\hline Stomach & 300 & 13.39 \\
\hline Kidney & 299 & 13.34 \\
\hline Heart & 289 & 12.90 \\
\hline Liver & 278 & 12.41 \\
\hline Large intestine & 90 & 4.02 \\
\hline Gallbladder & 56 & 2.50 \\
\hline Bladder & 50 & 2.23 \\
\hline Tri-jiao & 27 & 1.20
\end{tabular}

In the anti-yellowing formulas, warming was the main characteristic. Sweet, pungent and bitter were the main flavours. Based on this result, we can infer that nature and flavour tended to be gentle. A quarter of the drugs were associated with spleen channel tropism. Then, we connected nature, flavour and channel tropism to make a Sankey diagram. We intuitively found that the main nature and flavour tended to coordinate the intestines and stomach. 
Figure 2. Sankey diagram of the anti-yellowing formulas' nature, flavour and channel tropism

3.1.3 Formula rules of anti-yellow formulas

We extracted the most commonly used drug pairs in anti-yellowing formulas and sorted them by frequency (Table 4). Panax ginseng C. A. Mey, Poria cocos (Schw. Wolf, Atractylodes macrocephala Koidz., Angelica sinensis (Oliv.) Diels, Paeonia lactiflora Pall. and Astragalus membranaceus (Fisch.) Bge. merged in pairs formed the commonly used TCM pairs of anti-yellowing formulas. The associated drug network shows that the core of the medicine is $P$. ginseng C. A.Mey, followed by $A$. macrocephala Koidz., $A$. sinensis (Oliv.) Diels and $P$. lactiflora Pall.

Table 4. CommonlyTCM pairs of anti-yellow formulas

NO. TCM Pairs

Frequency

1 Panax ginseng C. A.Mey \& Poria cocos (Schw.)Wolf 28

$2 \quad$ Panax ginseng C. A.Mey \& Atractylodes macrocephala Koidz. 27

3 Atractylodes macrocephala Koidz. \& Poria cocos (Schw.)Wolf 23

4 Panax ginseng C. A.Mey \& Atractylodes macrocephala Koidz.\& Poria cocos (Schw.)Wolf 19

5 Panax ginseng C. A.Mey \& Astragalus membranaceus (Fisch. ) Bge. 17

$6 \quad$ Atractylodes macrocephala Koidz. \& Angelica sinensis (Oliv.) Diels 17

$7 \quad$ Angelica sinensis (Oliv.) Diels \& Paeonia lactiflora Pall. 17

$8 \quad$ Panax ginseng C. A.Mey \& Angelica sinensis (Oliv.) Diels 17

9 Panax ginseng C. A.Mey \& Paeonia lactiflora Pall. 16

10 Atractylodes macrocephala Koidz. \& Paeonia lactiflora Pall. 16

Figure 3. Associated TCM network

3.1.4 Empirical formulas and new formula

A correlation degree of 5 and penalty degree of 2 were set in the system to carry out hierarchical clustering analysis and extract new formula combinations.

According to the 2015 edition of the list of names of used cosmetic ingredients, the new formulas NO.2 and NO.3 contained non-cosmetic beneficial ingredients, so 3 empirical formulas (EFs) and the first new formula (NF) were retained (Table 5).

Table 5. Empirical formulas and new formula \additional file『

3.2 Biochemical efficacy tests results 


\subsubsection{Flavone content}

The results of the determination of the total flavonoid content of the four groups of lyophilized powders showed that the total flavonoid content of the new formula extract calculated by the software program was approximately three to six times higher than that of the three groups of experimental formulas.

Figure 4. Total flavonoid content of the lyophilized formula extract powder

\subsubsection{DPPH radical scavenging activity}

The results of the determination of DPPH free radical scavenging ability (Figure 5) showed that the new formula had the highest DPPH free radical scavenging activity, followed by the Xiaojianzhong decoction, the activity of which was significantly higher than that of the Sijunzi decoction and the angelica blood-enriching decoction.

Figure 5. DPPH scavenging activity

\subsubsection{Non-enzymatic glycosylation inhibiting activity}

In the in vitro inhibition of non-enzymatic glycosylation experiment (Figure 6), the new formula showed the most potent inhibition of glycosylation at the three concentrations tested, and the effect was the same as that of the clinically applied drug aminoguanidine at $1.0 \%$ and more effective than that at $2.0 \%$.

In the above experiments, among the four groups of freeze-dried powder extracts, the new formula had the best total flavonoid content, glycosylation inhibition activity, and DPPH free radical scavenging ability. According to these results, the optimal composition of the four groups of formula extracts was determined to be the new formula calculated systematically.

Figure 6. Non-enzymatic glycosylation inhibition activity

\subsection{Process optimization}

\subsubsection{Single factor experiment results}

By a single factor test, the new formula composition was extracted when the concentration of the ethanol solution was $60 \%-90 \%$, ultrasonication time was 20 min-40 min, material-liquid ratio was 1:30, and the flavonoid yield was the highest.

\subsubsection{Orthogonal test results}

In the orthogonal experiment, the flavonoid content and inhibition of the non-enzymatic glycosylation reaction were used as reference indexes, and $30 \%$ and $70 \%$ weights were included in the comprehensive score, respectively (Table 6).

$Y=\left(Y_{1} / Y_{1 M A X}{ }^{0} 0.3+Y_{2} / Y_{2 M A X} * 0.7\right) \star 100$

Table 6. Extraction process optimization for the orthogonal experiment 
NO. Factors

$\mathrm{Y}_{1} \quad \mathrm{Y}_{2}$

Total

$\begin{array}{lll}\begin{array}{l}\text { Material-liquid } \\ \text { ratio }\end{array} & \text { Time } & \begin{array}{l}\text { Ethanol } \\ \text { concentration(\%) }\end{array} \\ & (\min ) & \end{array}$

Flavonoid

AGEs

Y

(\%)

\begin{tabular}{|c|c|c|c|c|c|c|}
\hline 1 & $1: 20$ & 20 & 70 & 10.95 & 45.60 & 56.43 \\
\hline 2 & $1: 20$ & 30 & 80 & 9.04 & 78.69 & 77.74 \\
\hline 3 & $1: 20$ & 40 & 90 & 8.94 & 87.10 & 83.92 \\
\hline 4 & $1: 30$ & 20 & 80 & 12.03 & 78.47 & 83.53 \\
\hline 5 & $1: 30$ & 30 & 90 & 12.98 & 92.24 & 95.87 \\
\hline 6 & $1: 30$ & 40 & 70 & 8.45 & 55.15 & 58.70 \\
\hline 7 & $1: 40$ & 20 & 90 & 15.05 & 84.89 & 94.42 \\
\hline 8 & $1: 40$ & 30 & 70 & 6.91 & 61.95 & 60.79 \\
\hline 9 & $1: 40$ & 40 & 80 & 10.40 & 83.27 & 83.92 \\
\hline K1 & 218.09 & 234.38 & 175.92 & & & \\
\hline $\mathrm{K} 2$ & 238.10 & 234.40 & 245.19 & & & \\
\hline K3 & 239.13 & 226.54 & 274.22 & & & \\
\hline $\mathrm{R}$ & 21.04 & 7.86 & 98.30 & & & \\
\hline
\end{tabular}

Optimal conditions: A3, B2, C3

The optimal extraction conditions were $90 \%$ ethanol solution, a material-liquid ratio of 1:40, and ultrasonic extraction for $30 \mathrm{~min}$. The solid-liquid ratio of 1:30 or 1:40 and different ultrasonication times had little effect on the flavonoid extraction rate and on the inhibition of the non-enzymatic glycosylation reaction. When the volume fraction of ethanol reached $90 \%$, the end product could not be uniformly added to the cosmetic formula. Based on the experimental results and practical application, the final extraction conditions were determined to be as follows: ethanol solution with a volume fraction of $80 \%$, a material-liquid ratio of $1: 30$, and ultrasonic extraction for 30 min.

\subsection{Safety tests result}

\subsubsection{HET-CAM test result}

The positive control chicken embryo showed severe bleeding, blood vessel dissolution, and clotting, while the negative control chicken embryo showed no significant change, and the test system was established. $E S=8$, and $5 \%$ of the new formula compositions showed no eye irritation effect.

Figure 7. HET-CAM test results showed that the new formula had no eye irritation effect.

3.4.2 In vitro 3T3 NRU phototoxicity result 
The OD540 values in the solvent control were all greater than 0.4 . The positive control was established with IC50 $(+U V)=0.6022 \mu \mathrm{g} / \mathrm{mL}$, IC50 (-UV) $=29.91 \mu \mathrm{g} / \mathrm{mL}, \mathrm{PIF}=49.85$, and MPE $=0.551$, which were in line with OECD standards. The $\mathrm{IC}_{50}$ of the tested samples was higher than $1000.0 \mathrm{~g} / \mathrm{mL}$, the PIF value was significantly higher than 2.0 and less than 5.0, and the MPE value was higher than 0.15 . According to the test evaluation criteria, the potential phototoxicity of the tested samples was predicted, and the specific results are shown in Table 7.

Table 7. In vitro 3T3 NRU phototoxicity test result. It does not show cytotoxicity when it reaches the highest allowable concentration value $(1000.0 \mu \mathrm{g} / \mathrm{mL}) ;+$ : Expected phototoxicity; - : Expected no phototoxicity.

\begin{tabular}{llllll} 
Control/ Test & \multicolumn{2}{l}{$\mathrm{IC}_{50} \rrbracket \mu \mathrm{g} / \mathrm{mL} \square$} & PIF & MPF & phototoxicity \\
\cline { 2 - 3 } & No light & Light & & & \\
\hline CPZ & 19.71 & 0.85 & 23.16 & 0.53 & + \\
\hline Sample & $>1000.0$ & 238.51 & 4.19 & 0.25 & +
\end{tabular}

3.4.3 Multiple skin irritation test result

Table 8. The results of multipled skin irritation test. 
Day Number of animals Irritation response score

\begin{tabular}{|c|c|c|c|c|c|c|c|}
\hline & & \multicolumn{3}{|l|}{$\mathrm{NF}$} & \multicolumn{3}{|l|}{ Blank } \\
\hline & & Erythema & Oedema & Totle & Erythema & Oedema & Totle \\
\hline 1 & 4 & $0 / 4$ & 0 & 0 & 0 & 0 & 0 \\
\hline 2 & 4 & $0 / 4$ & 0 & 0 & 0 & 0 & 0 \\
\hline 3 & 4 & $0 / 4$ & 0 & 0 & 0 & 0 & 0 \\
\hline 4 & 4 & $0 / 4$ & 0 & 0 & 0 & 0 & 0 \\
\hline 5 & 4 & $0 / 4$ & 0 & 0 & 0 & 0 & 0 \\
\hline 6 & 4 & $0 / 4$ & 0 & 0 & 0 & 0 & 0 \\
\hline 7 & 4 & $0 / 4$ & 0 & 0 & 0 & 0 & 0 \\
\hline 8 & 4 & $0 / 4$ & 0 & 0 & 0 & 0 & 0 \\
\hline 9 & 4 & $0 / 4$ & 0 & 0 & 0 & 0 & 0 \\
\hline 10 & 4 & $0 / 4$ & 0 & 0 & 0 & 0 & 0 \\
\hline 11 & 4 & $0 / 4$ & 0 & 0 & 0 & 0 & 0 \\
\hline 12 & 4 & $0 / 4$ & 0 & 0 & 0 & 0 & 0 \\
\hline 13 & 4 & $0 / 4$ & 0 & 0 & 0 & 0 & 0 \\
\hline 14 & 4 & $0 / 4$ & 0 & 0 & 0 & 0 & 0 \\
\hline
\end{tabular}

Average scores of per animal and per day 0

Irritation level

No irritation was observed

3.4.4 24-hour human body closed patch test

Table 9. 24-hour human body closed patch test result 


\begin{tabular}{|c|c|c|c|c|c|c|c|c|}
\hline $\begin{array}{l}\text { sample } \\
\text { name }\end{array}$ & $\begin{array}{l}\text { Observation } \\
\text { time } \rrbracket h \rrbracket\end{array}$ & $\begin{array}{l}\text { Sample } \\
\text { concentration } \\
\varangle \% \bigotimes\end{array}$ & $\begin{array}{l}\text { Fall } \\
\text { off }\end{array}$ & $\begin{array}{l}\text { negative } \\
\text { 『०ठ }\end{array}$ & $\begin{array}{l}\text { suspicious } \\
\mathbb{1}_{1 \rrbracket}\end{array}$ & $\begin{array}{l}\text { Weak } \\
\text { positive } \\
₫ 2 \rrbracket\end{array}$ & $\begin{array}{l}\text { Strong } \\
\text { positive } \\
\bigotimes 3 \rrbracket\end{array}$ & 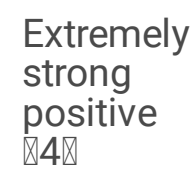 \\
\hline \multirow{3}{*}{$\begin{array}{l}\text { Gel } \\
\text { matrix }\end{array}$} & 0.5 & \multirow[t]{3}{*}{-} & 0 & 27 & 1 & 0 & 0 & 0 \\
\hline & 24 & & 0 & 28 & 0 & 0 & 0 & 0 \\
\hline & 48 & & 0 & 28 & 0 & 0 & 0 & 0 \\
\hline \multirow{3}{*}{$\begin{array}{l}\text { Adding } \\
\text { gel }\end{array}$} & 0.5 & \multirow[t]{3}{*}{5} & 0 & 27 & 1 & 0 & 0 & 0 \\
\hline & 24 & & 0 & 28 & 0 & 0 & 0 & 0 \\
\hline & 48 & & 0 & 28 & 0 & 0 & 0 & 0 \\
\hline
\end{tabular}

According to the results in Table 7-9 and the interpretation of the results in the cosmetic hygiene code (2007 edition), the gel matrix and the gel with $5 \%$ composition have no adverse reactions with human skin.

No eye irritation effect of the new composition was detected by testing the chicken chorioallantoic membrane. The phototoxicity test of 3T3 determined that the new structure may have phototoxicity and should not be used in sunscreen products. Through several skin irritation tests, it was determined that the original composition was not irritating or corrosive to mammalian skin. It was determined that the new form had no adverse reaction with human skin by the human closed patch test. Based on the above safety test results, the new formula composition was determined to be safe for the human body and can be used as a cosmetic raw material.

\section{Discussion}

\subsection{New formulas do not fully comply with traditional formulating principles}

Sweet and bitter drugs are the main five drugs that make up the anti-yellowing formula. Sweet medicines can replenish energy and relieve, nourish, and neutralize. They are mostly used to treat weakness of righteousness and reconcile medicines; spicy medicines have a strong ability to diverge, have the effect of qi and blood, disperse the table, are mostly used to treat the symptoms of block and wet spleen, and have heat clearing and detoxifying effects. It is inferred that the medicines used for treatment of dark facial yellowing tend to be solid, relieve stasis, and detoxify.

The formulas issued by doctors of TCM are based on the solid basic theory of TCM and rich clinical experience. Therefore, we reviewed the new formulas obtained from analysis of the theoretical applications of compatible formulas among Chinese medicines. The drug composition of the new formula is not in line with the theoretical applications of compatible formulas among well-known Chinese medicines, but its role is consistent with the theory underlying the use of TCM to treat the problem of yellow complexion. 
In addition, most formulas were created in ancient times, when yellow complexion was a sign of malnutrition. However, malnutrition has been replaced by AGEs in modern day. The most typical example is patients with diabetes, which may be understood as another form of overnutrition. Regarding the relationship between yellow complexion and nutrition, it is speculated that the new formula and its secondary metabolites may have an effect on the intestinal flora.

\subsection{Empirical formulas are still worth to explore on anti-yellow}

The three EFs were obtained through the analysis of similar formulas. As far as possible, high-frequency medicinal materials and high-frequency medicinal pairs were included. The combinations were the closest matches to the trends of all the formulas. Sijunzi decoction ${ }^{[24-26]}$ is a tonic supplement that nourishes qi and strengthens the spleen. Danggui Buxue decoction ${ }^{[27-29]}$ is a tonic supplement that has a significant effect on blood nourishment. Xiaojianzhong decoction ${ }^{[30]}$ is a warming agent that is used for tonifying deficiency and warming in the abdomen and is used to treat liver and spleen deficiency syndrome. The three EFs focus on invigorating qi, invigorating blood, and alleviating the deficiency. Biochemical experiments show that the practical formula Xiaojianzhong decoction has the best effect in the treatment of yellowing, second only to the new formula.

\subsection{New formula was effective at a range of biochemical experiments}

The new formula was also tested by volunteers at a low level after the safety test. A 5\% concentration was added to the mask matrix. After mask application, the $B^{*}$ value, which represents the yellow-blue phase of the skin, was significantly reduced. We will continue in vivo testing in the future.

\subsection{This method could be used on application of cosmetic formula and development of cosmetic raw materials}

Chinese medicine is a highly practical subject. Due to the large number of studies and classics and because it is not easy to save or retrieve the formulas, there is valuable information that has not yet been obtained.

Documents can be digitized for easy storage and retrieval. To date, a great deal of work has been done in the field of Chinese medicine, and a large number of databases have been established. A large amount of hidden knowledge contained in these databases has not been fully extracted and utilized. The development of datamining technology will undoubtedly be one of the directions for research on TCM in the future.

There are thousands of TCM cosmetic formulas based on Chinese herbal medicine-flavoured ingredients produced in China. With the rapid development of China's national strength and the prosperity of the beauty and personal care products market, guided by the philosophy of TCM (both internal and external) and the multitarget synergies, prevention and treatment methods for TCM cosmetology, especially via expanded application of past classics, the market share has become extremely impressive.

\section{Conclusion}

This study explored and analysed the internal laws of TCM for treatment of yellow complexion and, on this basis, developed a plant combination extract material with an anti-yellowing effect on skin. 
For the first time, this study found a new plant composition suitable for use as a cosmetic raw material from a TCM formula and preliminarily explored the methodology for the development of this composition. This study provides a scientific theoretical basis and material selection support for the application of TCM formulas in cosmetics and provides ideas for the modern-day development and application of TCM.

\section{Abbreviations}

AGEs

Advanced glycosylation end products

TCM

Traditional Chinese medicine

$\mathrm{DPPH}$

2,2-diphenyl-1-picrylhydrazyl

BSA

Bovine serum albumin

PBS

Phosphate-buffered saline

SDS

Sodium dodecyl sulphate

CPZ

Chlorpromazine hydrochloride

$\mathrm{EF}$

Empirical formula

$\mathrm{NF}$

New formula

\section{Declarations}

Ethics approval and consent to participate

Not applicable.

Consent for publication

Not applicable.

Availability of data and materials

The datasets used during the current study available from the corresponding author on reasonable request.

Competing interests

The authors declare that they have no competing interests.

Funding 
This work was supported by the Scientific Research Project of Beijing Educational Committee (KM202010011009) and Beijing Excellent Talent Training Project-Young Individuals (2018000020124G032), which supported to Pr. Fan YI. This work also supported by the Acupuncture-Moxibustion and Tuina (KLC-2017ZD2), which supported to Pr. Tuya Bao.

Authors' contributions

SY WANG and Fan YI conceived and designed the study; SY WANG performed the experiments; YM DONG, H MENG, L LI and TY BAO substantively revised it. All authors read and approved the final manuscript.

Acknowledgements

The authors thank Pr. Xisheng SANG and Pr. Yanyan ZHOU, Heilongjiang University Of Chinese Medicine, for they help in interpreting the significance of the results of this study. Thanks to Dr. Wang, Institute of acupuncture and moxibustion, CACMS, for his guidance and help in the retrieval process.

Authors' Information

Affiliations

1.Key Laboratory of Cosmetic, China National Light Industry, Beijing Technology and Business University『Beijing 100048هChina

Corresponding author

Correspondence to Fan YI.

\section{References}

1. YU Y, CAI M. ZHOU L, et al. Study on the aging and yellowing properties of sebum-soiled cotton fabrics [J]. Fibers \& Polymers, 17(2): 305-9.

2. BA?ULS J, ARRIBAS P. BERBEGAL $L$, et al. Yellow and orange in cutaneous lesions: clinical and dermoscopic data [J]. Journal of the European Academy of Dermatology \& Venereology, 29(12): 2317-25.

3. KNUDSEN A. Measurement of the Yellow Colour of the Skin as a Test of Hyperbilirubinemia in Mature Newborns [J]. Acta Paediatr. 1991;79(12):1175-81.

4. GRAD SC, MURESAN I DUMITRASCUDL. Generalized Yellow Skin Caused by High Intake of Sea Buckthorn [J]. Forschende Komplementu00e4rmedizin, 19(3): 153-6.

5. LOGAN I T, LOGAN RA. The color of skin: yellow diseases of the skin, nails, and mucosa [J]. CLINICS IN DERMATOLOGY, 2019, 37(580 - 90.

6. FURQAN S, KAMANI L. JABBAR A. Skin manifestations in diabetes mellitus [J]. 2014, 26(1): 46.

7. DYER D G, DUNN J A, THORPE S R, et al. Accumulation of Maillard Reaction Products in Skin Collagen in Diabetes and Aging [J]. Journal of Clinical Investigation. 1993;91(6):2463.

8. SÉROUL P, CAMPICHE R, GOUGEON S, et al. An image-based mapping of significance and relevance of facial skin colour changes of females living in Thailand [J]. International Journal of Cosmetic Science, 2020. 
9. TAN K W. STEPHEN I D. Colour detection thresholds in faces and colour patches [J]. Perception, 42(7): 73341.

10. KOULIS C, WATSON A, GRAY S, et al. Linking RAGE and Nox in diabetic micro- and macrovascular complications [J]. Diabetes \& Metabolism, 41(4): 272-81.

11. GEORGAKOPOULOU E, TSIMARATOU K, EVANGELOU K, et al. Specific lipofuscin staining as a novel biomarker to detect replicative and stress-induced senescence. A method applicable in cryo-preserved and archival tissues [J]. Aging, 5(1): 37-50.

12. BRUNK U T. TERMAN A. Lipofuscin: mechanisms of age-related accumulation and influence on cell function [J]. Free Radical Biology \& Medicine, 33(5): 611-9.

13. YING LIANG. CHUAN, et al. Matrix metalloproteinase 9 induces keratinocyte apoptosis through FasL/Fas pathway in diabetic wound [J].

14. VERMA N, MANNA S K. Advanced glycation end products (AGE) potentiates cell death in p53 negative cells via upregulaion of NF-kappa B and impairment of autophagy [J]. Journal of Cellular Physiology, 2017.

15. Effects of advanced glycation. end-products on skin keratinocytes by NF-kB activation [J]. Chinese Journal of Trauma, 2006.

16. YOKOTA M, TOKUDOME Y. The Effect of Glycation on Epidermal Lipid Content, Its Metabolism and Change in Barrier Function [J]. Skin Pharmacology \& Physiology, 231 - 42.

17. YOKOTA M, MASAKI H, OKANO Y, et al. Effect of glycation focusing on the process of epidermal lipid synthesis in a reconstructed skin model and membrane fluidity of stratum corneum lipids [J]. Dermatoendocrinology, 2017, 9(1).

18. MOU K, LIU W. HAN D, et al. HMGB1/RAGE axis promotes autophagy and protects keratinocytes from ultraviolet radiation-induced cell death [J]. Journal of Dermatological Science, 85(3): 162-9.

19. ZHAO J, ZHONG A, FRIEDRICH E E, et al. S100A12 Induced in the Epidermis by Reduced Hydration Activates Dermal Fibroblasts and Causes Dermal Fibrosis [J]. Journal of Investigative Dermatology, 2016, 137(3).

20. HEGAB Z, GIBBONS S. NEYSES L, et al. Role of advanced glycation end products in cardiovascular disease [J]. World Journal of Cardiology, 04): 4-16.

21. ANNA S, EL?BIETA B, EWA T-G, et al. Melanin and lipofuscin as hallmarks of skin aging [J]. Advances in Dermatology \& Allergology/post囚py Dermatologii I Alergologii, 2(97-103.

22. LEE E J, KIM J Y, OH SH. Advanced glycation end products (AGEs) promote melanogenesis through receptor for AGEs [J]. Scientific Reports, 2016, 6(27848.

23. Oxidative stress-induced HMGB1 release from melanocytes: a paracrine mechanism underlying the cutaneous inflammation in vitiligo [J].

24. DAI L, ZHOU W, WANG M, et al. Efficacy and safety of Sijunzi Decoction for chronic fatigue syndrome with spleen deficiency pattern: study protocol for a randomized, double-blind, placebo-controlled trial [J]. Ann Transl Med. 2019;7(20):587.

25. TIAN G, WU C, LI J, et al. Network pharmacology based investigation into the effect and mechanism of Modified Sijunzi Decoction against the subtypes of chronic atrophic gastritis [J]. Pharmacol Res, 2019, $144(158-66$.

26. GAO B, WANG R, PENG Y, et al. Effects of a homogeneous polysaccharide from Sijunzi decoction on human intestinal microbes and short chain fatty acids in vitro [J]. J Ethnopharmacol, 2018, 224(465 - 73. 
27. LIU Y, LIX, LI A, et al. UHPLC Q-Exactive MS-based spleen metabolomics and lipidomics to explore the effect mechanisms of Danggui Buxue Decoction in anemia mice [J]. J Pharm Biomed Anal, 2020, 185(113234.

28. FANG L, CHENG C, HWANG T, et al. Danggui buxue tang inhibits 2,4-dinitrochlorobenzene: induced atopic dermatitis in mice [J]. Evid Based Complement Alternat Med, 2015, $2015(672891$.

29. MIAO X, XIAO B. SHUI S, et al. Metabolomics analysis of serum reveals the effect of Danggui Buxue Tang on fatigued mice induced by exhausting physical exercise [J]. J Pharm Biomed Anal. 2018;151:301-9.

30. DU X. [Essence of professor SHI Xin-de's experience in application of persicae ramulus-associated prescriptions] [J]. Zhongguo Zhong Yao Za Zhi. 2014;39(13):2569-72.

\section{Figures}

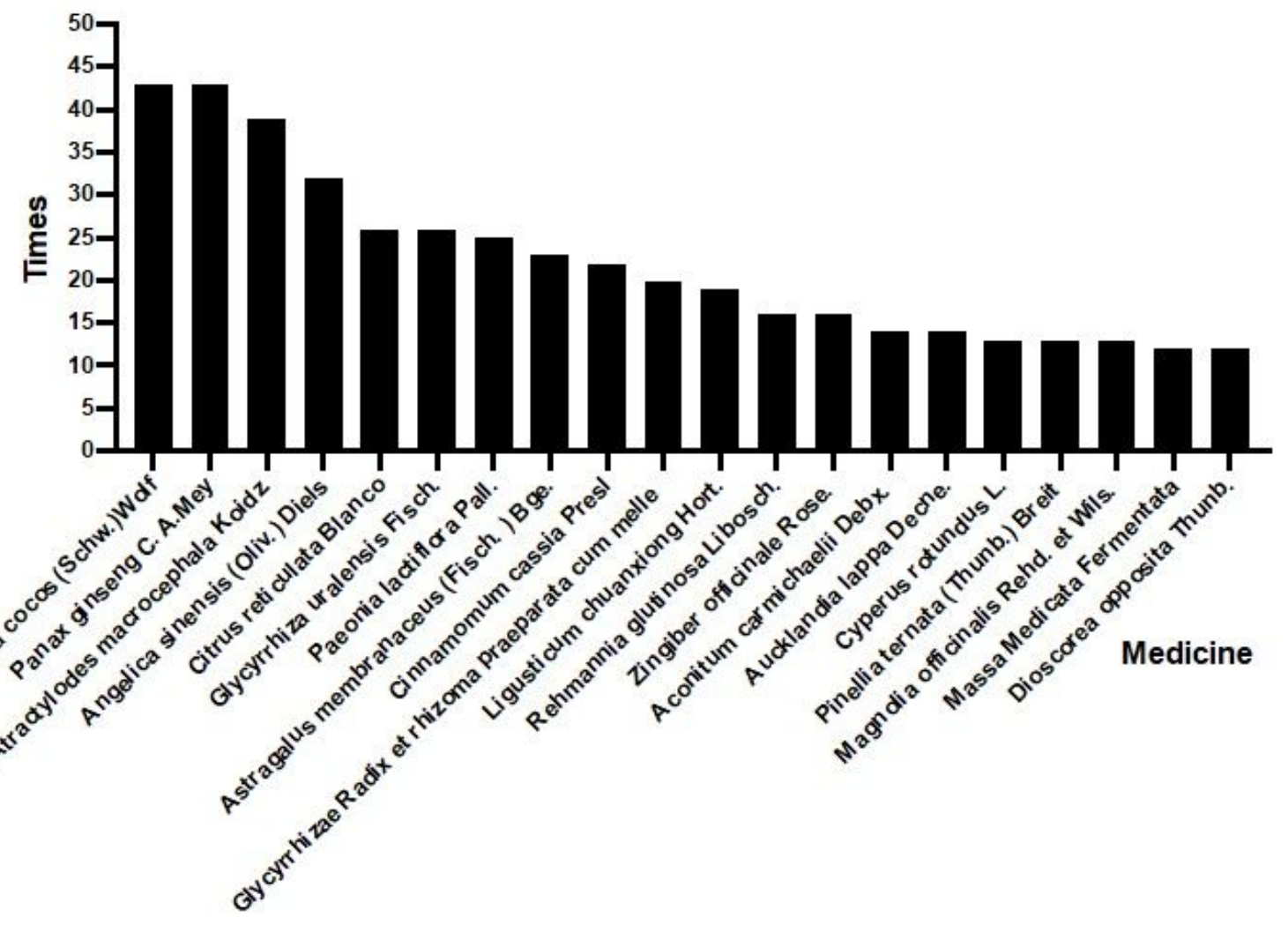

Figure 1

High-frequency TCMs in anti-yellowing formulas. Approximately half of the formulas contained four herbs: Indian bread (Poria), Ginseng, Atractylodes and Chinese angelica. 


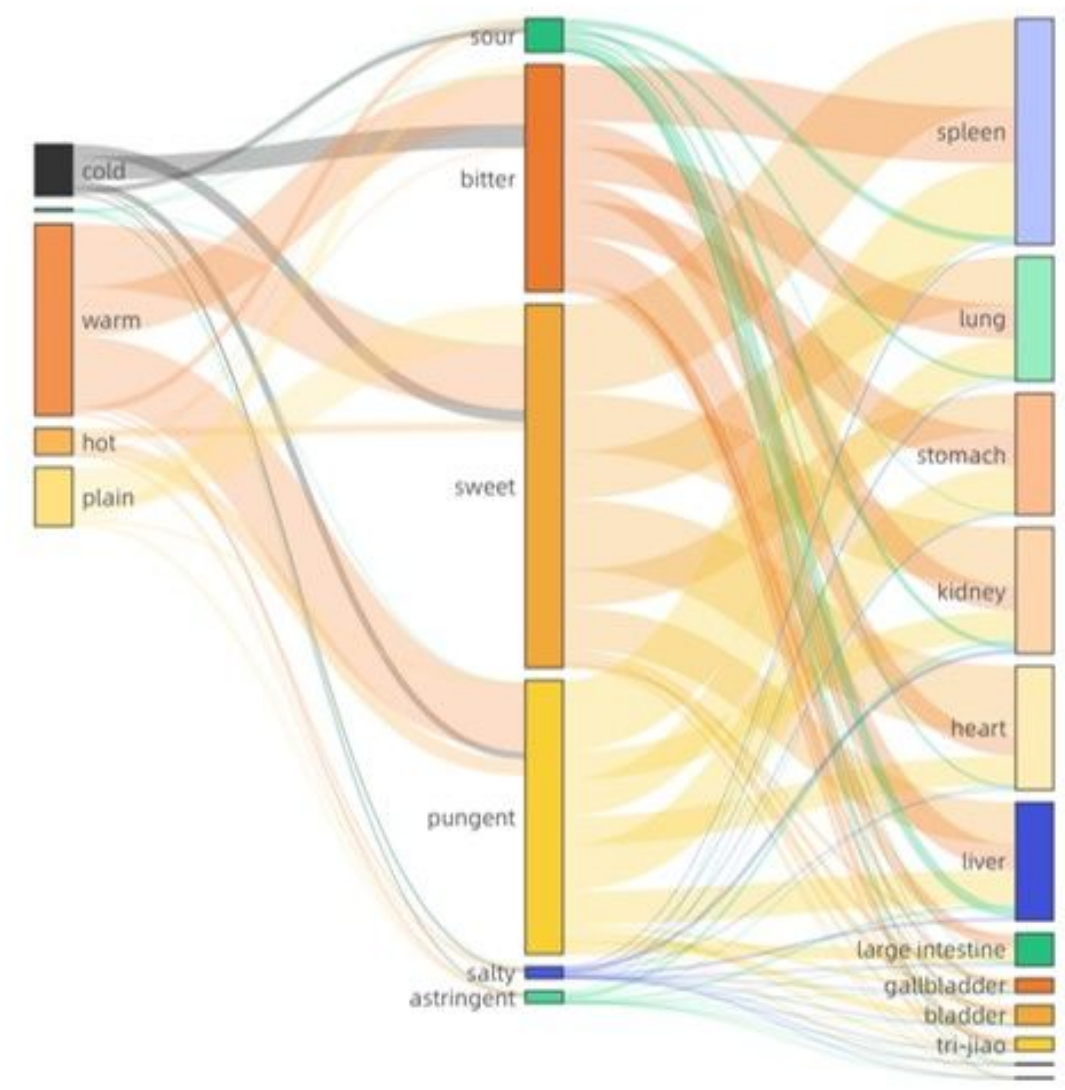

\section{Figure 2}

Sankey diagram of the anti-yellowing formulas' nature, flavour and channel tropism

Astragalus membranaceus (Fisch.) Bge.

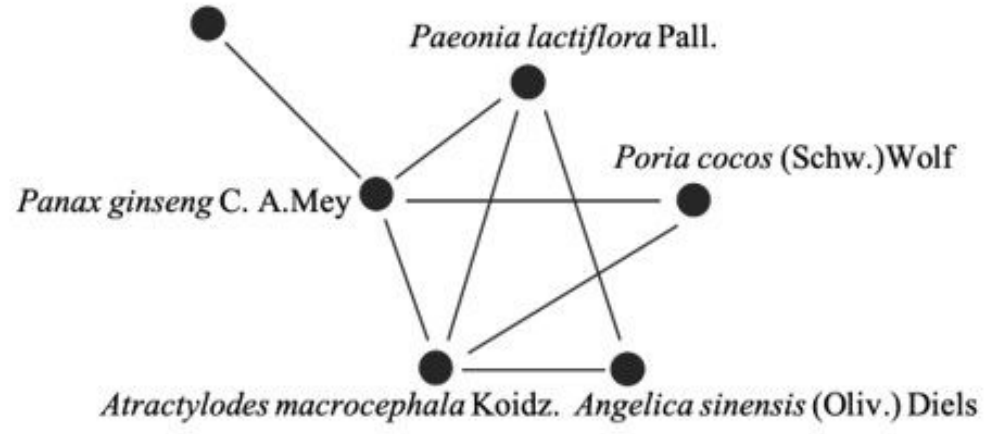

\section{Figure 3}

Associated TCM network 


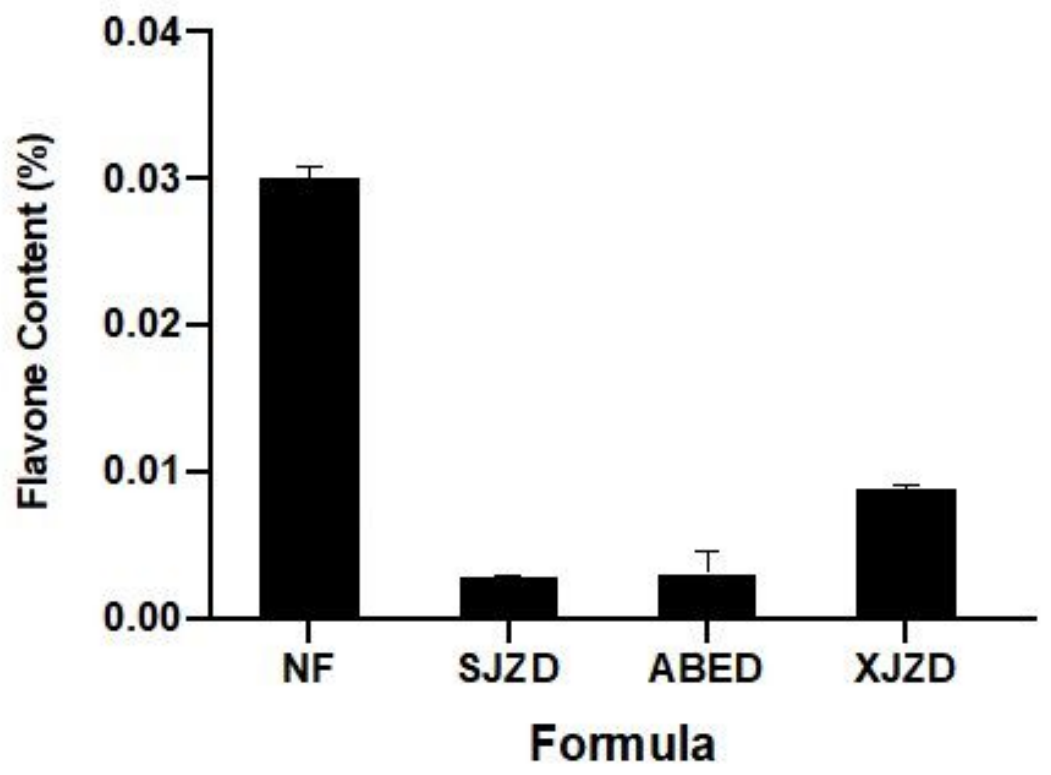

Figure 4

Total flavonoid content of the lyophilized formula extract powder

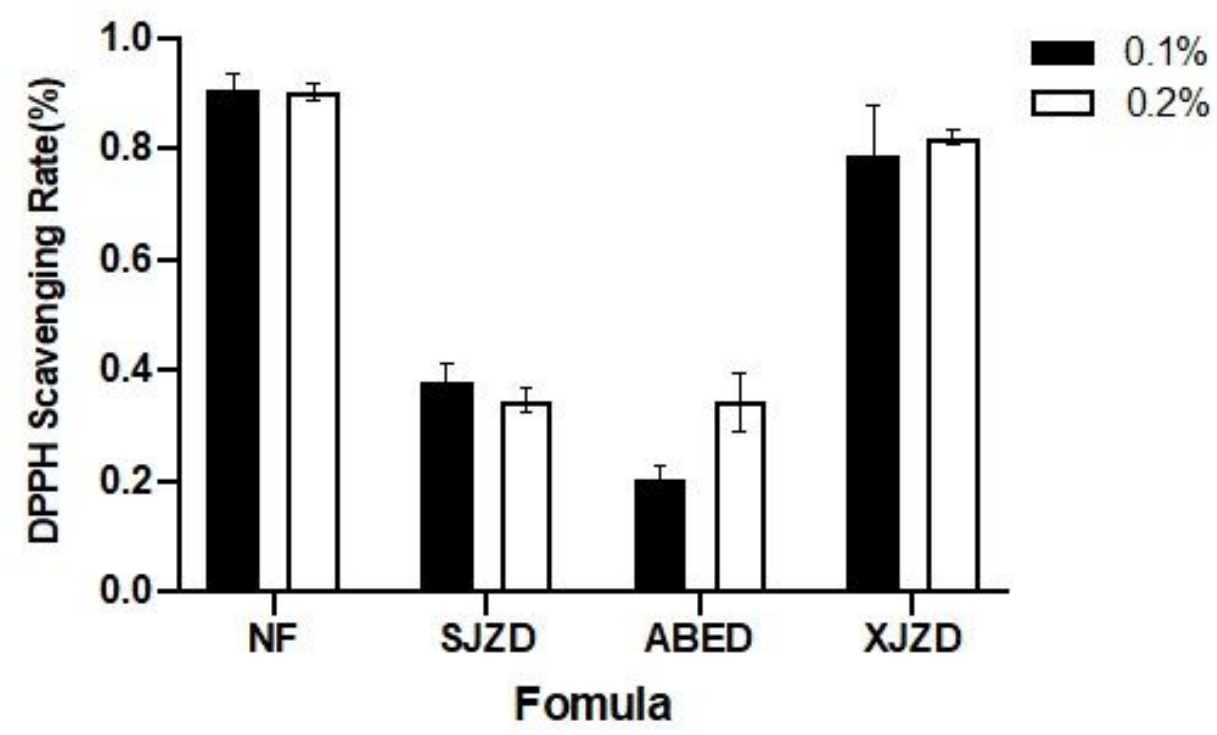

Figure 5

DPPH scavenging activity 


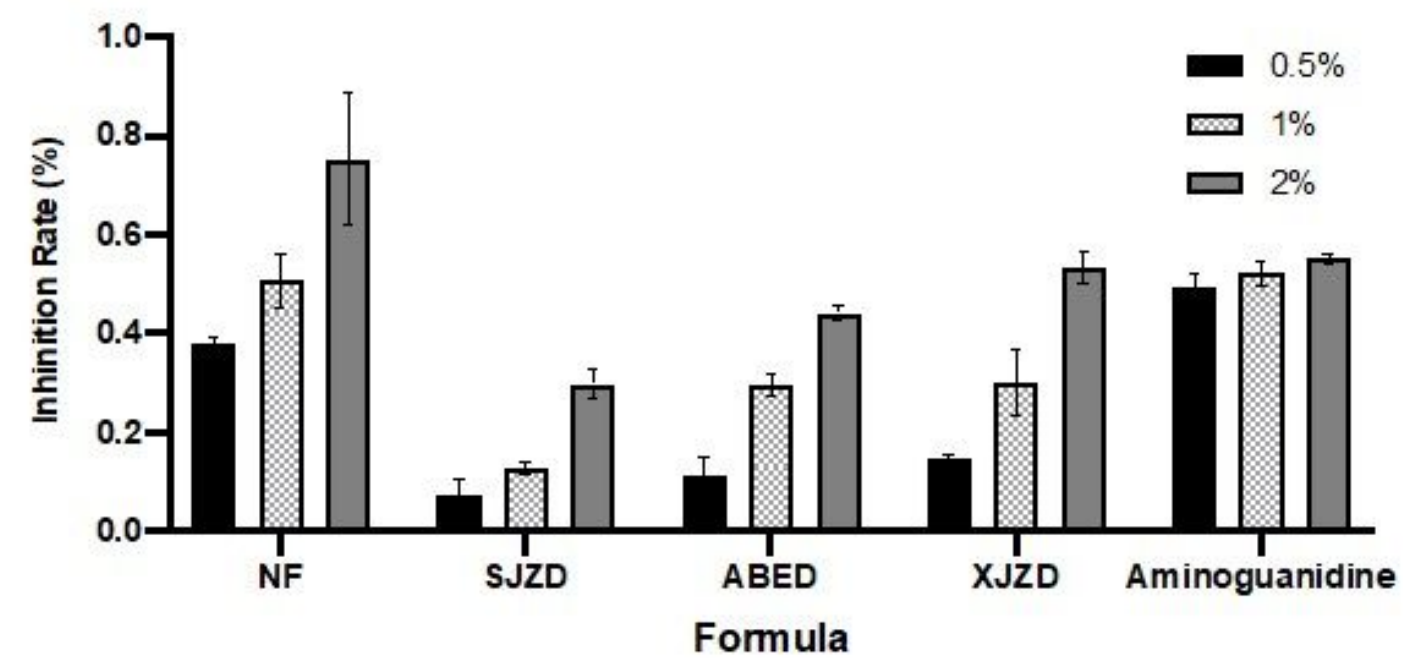

Figure 6

Non-enzymatic glycosylation inhibition activity

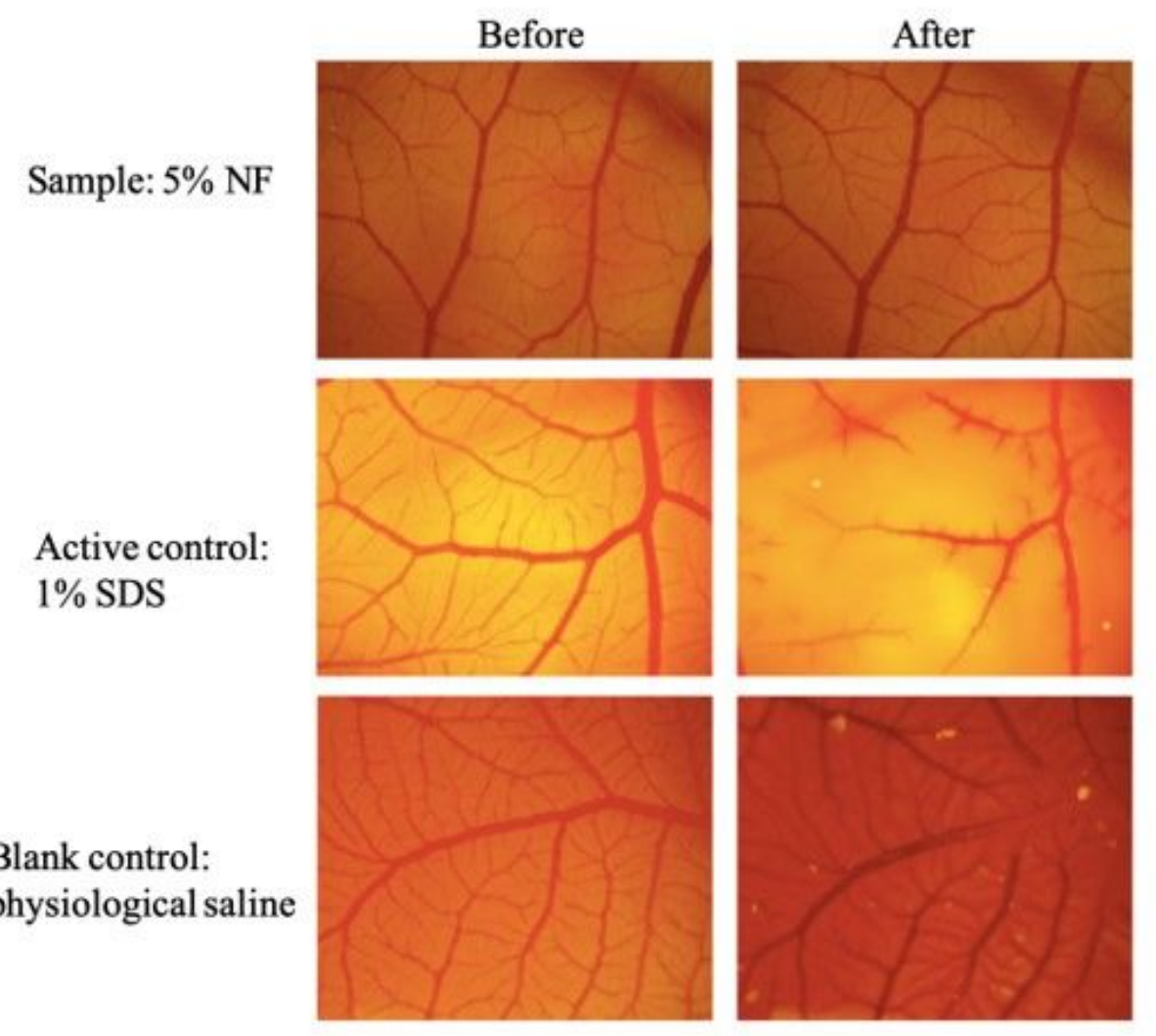


Figure 7

HET-CAM test results showed that the new formula had no eye irritation effect.

\section{Supplementary Files}

This is a list of supplementary files associated with this preprint. Click to download.

- TOC.jpg 